[0212-7199 (2005) 22: 2: pp 85-87] ANALES DE MEDICINA INTERNA Copyright (C) 2005 ARAN EDICIONES, S.L.

AN. MED. INTERNA (Madrid) Vol. 22, N. ${ }^{\circ} 2$, pp. 85-87, 2005

\title{
Esteatohepatitis no alcohólica, el enigma de una mala evolucion
}

\author{
M. LÓPEZ-DIÉGUEZ PUERTA, V. CASAL ESTEBAN, F. J. BARBADO HERNÁNDEZ, \\ L. GIL GUERRERO, M. A. RODRÍGUEZ MOLINERO, J. LARRAURI, J. J. VÁZQUEZ \\ RODRÍGUEZ
}

Servicio de Medicina Interna. Hospital Universitario la Paz. Madrid

\author{
NONALCOHOLIC STEATOHEPATITIS, THE ENIGMA OF BAD PRO- \\ GRESS
}

\section{RESUMEN}

Hasta ahora poco se sabe acerca de la patogenia, los desencadenantes y en particular de la evolución y terapia de la esteatohepatitis no alcohólica (EHNA). Incluída en el amplio espectro de enfermedades del hígado graso no alcohólicas. Se ha considerado una enfermedad mayoritariamente benigna y asociada a alteraciones metabólicas muy prevalentes en la población general. Sin embargo no es raro que progrese a cirrosis, insuficiencia hepática o hepatocarcinoma que precise transplante hepático.

Presentamos el caso de una paciente que diagnosticamos de EHNA, al inicio con respuesta parcial y tardía al tratamiento para el sobrepeso, la HTA y la hiperlipidemia, pero con una evolución rápida a cirrosis hepática sin identificarse ningún factor desencadenante. Actualmente es candidata a transplante hepático.

Son necesarios estudios multicéntricos y controlados, con la misma definición de EHNA y objetivos comunes que aportarán información sobre datos diagnósticos y nuevas terapias para el manejo precoz de la enfermedad.

PALABRAS CLAVE: Esteatohepatitis no alcohólica. Evolución. Cirrosis

\begin{abstract}
Still, very little is known about the precise pathogenetic mechanisms, the triggering events and in particular, the evolution and treatment of non-alcoholic steatohepatitis (NASH). It is part of the broad spectrum of nonalcoholic fatty liver diseases (NAFLDS). Mainly, it has been reported as a benign disease, asociated with metabolic disorders commonly occurrence en the general population. Nevertheless, the syndrome can lead to cirrhosis, liver failure or hepatocellular carcinoma, requiring liver transplantation.

We present one pacient with diagnosis of NASH, who was treated initially for overweight, HTA and hyperlipaemia with incompleted response and who showed a quickly progress to cirrosis but no cause of liver descompensated disease could be identified. Currently she is at end-stage waitting a liver transplantation.

Controlled and multicentric studies with the same definition of NASH and the study end-points are needed, and will provide information about diagnosis features and novel therapies to early management of the disease.
\end{abstract}

KEY WORDS: Nonalcoholic steatohepatitis. Progress. Cirrosis.

López-Diéguez Puerta M, Casal Esteban V, Barbado Hernández FJ, Gil Guerrero L, Rodríguez Molinero MA, Larrauri J, Vázquez Rodríguez JJ. Esteatohepatitis no alcohólica, el enigma de una mala evolucion. An Med Interna (Madrid) 2005; 22: 85-87.

\section{INTRODUCCIÓN}

La esteatohepatitis no alcohólica es una entidad clínica que cada vez se diagnostica con más frecuencia, poco conocida. Descrita por primera vez por Ludwig (1), forma parte de un amplio espectro de alteraciones hepáticas, que abarcan desde el hígado graso hasta la cirrosis o el carcinoma hepatocelular (2).

La prevalencia es desconocida en la población general, pero se cree la tercera causa de enfermedad hepática crónica en nuestro medio. En los varones comienza a ser más frecuente el diagnóstico (3), así como en niños y adolescentes sobre todo obesos $(4,5)$, aunque hasta ahora ha sido más prevalente en el sexo femenino. Existe una fuerte asociación con facto- res de riesgo como son la obesidad, siendo más importante la distribución de la grasa que el propio IMC (6), la diabetes mellitus y muy especialmente la resistencia a la insulina (7).

Es muy relevante la hiperlipidemia donde juegan un mayor papel los triglicéridos que el colesterol (8). El síndrome hipermetabólico se ha relacionado hasta en un $20 \%$, la hipertensión arterial en un $30 \%$ y en menor proporción otos factores (hiperuricemia, cirugía para reducciones gástricas con anastomosis yeyuno-ileal, nutrición parenteral total, fármacos como la amiodarona, etc.) $(9,10)$.

Presentamos un caso de esteatohepatitis no alcohólica de desarrollo largo, complejo y peculiar, diagnosticado en una fase clínica precoz con buena respuesta inicial al tratamiento

Trabajo aceptado: 28 de octubre de 2004

Correspondencia: M. López-Diéguez Puerta. C/ Ofelia Nieto, 9, esc A, 1 izq.28039 Madrid. e-mail: arialdp5@hotmail.com 
de las alteraciones metabólicas, pero con una evolución a grave insuficiencia hepática sin un claro desencadenante.

\section{CASO APORTADO}

Mujer de 32 años de edad que tiene astenia desde hace 3 años, lesiones cutáneas en región malar y base del cuello, sequedad oral, tumefacción parotídea bilateral y artralgias en pequeñas articulaciones de pies y manos de carácter migratorio e intermitente. Remitida por médico de atención primaria con sospecha de lupus eritematoso sistémico y/o síndrome de Sjögren en noviembre 1999. En un estudio previo en otro centro hospitalario se realizan unos análisis en los que destacan anticuerpos antinucleares $1 / 80$ y una sialografía informada como parotiditis crónica y desestructuración del sistema ductal.

Antecedentes personales: asma bronquial hasta los 8 años de edad, apendicectomizada a los 10 años y un aborto espontáneo en 1998. No fumaba y la ingesta de alcohol se reducía a 10-20 g de forma ocasional. Actividad laboral: administrativo. Historia familiar sin interés.

Exploración física: sobrepeso con un IMC de 27 y una TA de 170/110, discreta hipertrofia parotídea, sin lesiones cutáneas ni signos inflamatorios articulares; el resto de la exploración física era normal.

Hemograma: 4,2 hematíes, 14,6 Hb, 41\% Hto, 97 VCM, 35 HCM, 408.000 plaquetas. Bioquímica: triglicéridos 1687 cifra con posterior descenso hasta noviembre del 2000 y brusca elevación en mayo del 2001; colesterol 269 (VLDL 62\% y HDL/LDL 19).GPT 74, GOT 50, GGT 400. Proteinograma: PT 5,5, albúmina 69\%, subunidad gamma 9\%. Inmunoelectroforesis: IgG 419. Anticuerpos antiLKM, ANA, antimitocondriales y antitiroideos negativos.

Radiografía de tórax normal; ecografía abdominal con hígado de tamaño y morfología normales, homogéneo sin LOES y con hiperecogenicidad fuerte y brillante que sugería esteatosis. Biopsia de glándulas salivares menores de labio inferior sin lesiones valorables.

Biopsia hepática con marcado ecográfico: fibrosis estrellada de los espacios porta que en ocasiones llega a unirlos entre sí sin llegar a producir nódulos de regeneración, la fibrosis diseca los sinusoides del lobulillo hepático llegando a adoptar un patrón intersticial. Algún linfocito sin apreciarse claros infiltrados, intensa esteatosis de predominio macrovesicular que afecta a más de un $70 \%$ de los hepatocitos (Figs. 1 y 2). Aisladamente focos de necrosis con exudado polimorfo inflamatorio y ocasionalmente Hialina de Mallory sin evidencia de pigmento férrico ni colestasis.

Espectrofotometría de absorción atómica/cámara de grafito: hierro en tejido hepático $1.167 \mathrm{mcg} / \mathrm{g}$ peso seco, no compatible por tanto con hemocromatosis. El estudio genético para las mutaciones C282Y y H63D que afectan al gen HFE fue negativo.

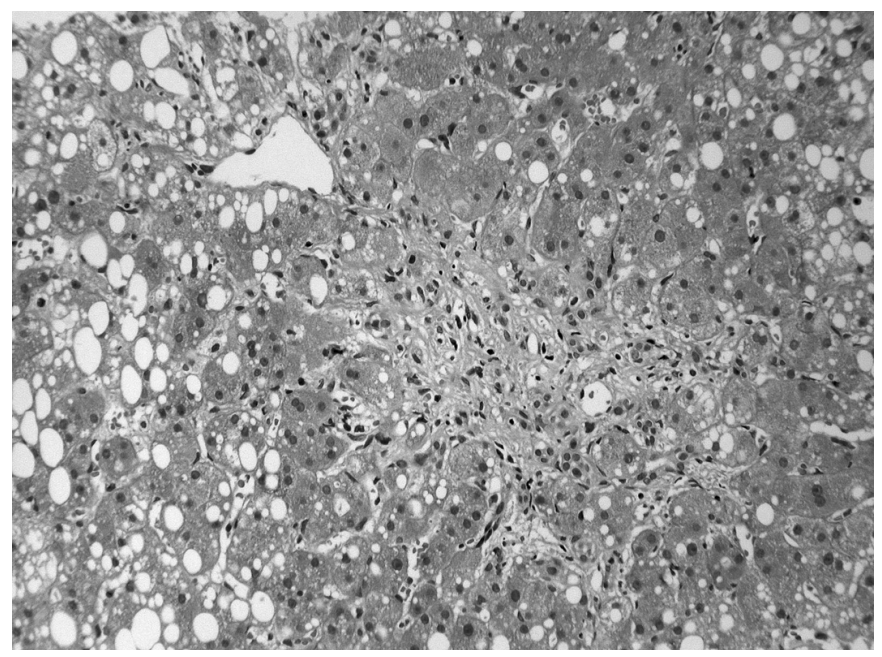

Fig. 1. Moderada fibrosis estrellada e intensa esteatosis (Mason x4)

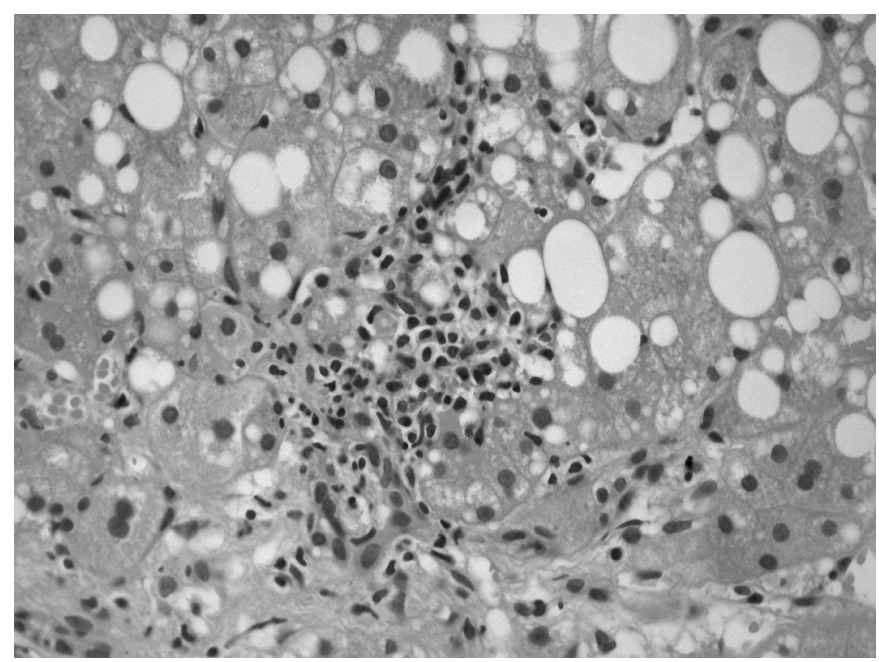

Fig. 2. Fibrosis perisinusoidal con intensa esteatosis y mínimo componente inflamatorio (Mason $\times 25$ ).

Un año después del comienzo de la clínica, tiene una rápida pérdida de peso de unos 10-12 kg, malestar general y exacerbación de la astenia. A la exploración física se objetivó una hepatomegalia gigante y dolorosa y los análisis mostraron una relación GOT/GPT superior a 3 cuando en la visita anterior no superaba 1,3, lo que se puede interpretar como progresión a la fibrosis hepática (11). Tenía leucocitosis con desviación a la izquierda de la serie blanca y anemia, persistía la macrocitosis y la hiperlipidemia con TG por encima de 1.000 $\mathrm{UI} / \mathrm{dl}$.

TC abdominal: gran hepatomegalia con imagen sugestiva de infiltración grasa. No existen LOES ni dilatación de radicales biliares intra ni extrahepáticos, vesícula biliar normal al igual que el resto de estructuras abdominales.

Se comenzó el tratamiento con ácido ursodesoxicólico y vitamina E junto con su terapia habitual para la HTA y la hiperlipidemia. Desde entonces seguida en consultas sin clara mejoría clínica, y en espera de trasplante hepático.

En noviembre de 2003 vuelve a ser ingresada con empeoramiento del estado general, ascitis y edemas en miembros inferiores. En ecografía abdominal se objetiva abundante líquido ascítico, gran hepatomegalia sin lesiones ocupantes de espacio, recanalización de la vena paraumbilical y colaterales en pared abdominal. Se practicó paracentesis evacuadora con extracción de diez litros.

La función hepática se mantuvo sin modificaciones respecto a los últimos controles y no presentó signo alguno de clínica infecciosa. Remitió la descompensación hidrópica y se dio el alta hospitalaria con buen estado general.

\section{DISCUSIÓN}

Nuestra paciente tenía inicialmente sobrepeso y síntomas inespecíficos, como cursa de forma habitual esta enfermedad (11), un año y medio después pierde $10-12 \mathrm{~kg}$ en poco tiempo coincidiendo con un empeoramiento de su sintomatología y la aparición de una hepatomegalia dura y dolorosa , con macrocitosis y posterior anemia y leucocitosis. Con una hiperlipidemia mixta mantenida a pesar del tratamiento combinado con estatinas y fibratos, que sólo se normalizó tras varios años de terapia, y con citolisis intensa y colestasis disociada además de una esteatosis importante con focos de necrosis e inflamación y fibrosis; todo ello sugestivo de esteato hepatitis alcohólica por ello reinterrogada en numerosas ocasiones sin admitir un consumo etílico significativo. Se preguntó por separado a 
los familiares que también negaban un abuso alcohólico y ella nunca presentó rasgo o signo que hiciera sospechar lo contrario, por lo tanto se establece el diagnóstico de esteatohepatitis no alcohólica.

Con este caso destaca la relevancia de un diagnóstico precoz en una entidad clínica muy prevalente, sin duda la patología hepática más común (12); hasta ahora considerada de evolución mayoritariamente benigna y sin embargo con un potencial de progresión hacia cirrosis muy notoria sobre todo si en la histología ya se evidencian claros signos de inflamación, (balonización de hepatocitos, hialina de Mallory, etc) $(13,14)$, No existen estudios prospectivos que aborden la historia natural de esta entidad de forma estricta, pero un gran número de cirrosis hepática hasta ahora clasificadas como criptogénicas han sido diagnosticadas finalmente como estea- tohepatitis no alcoholica.

Su fisiopatología es multifactorial y está implicado el síndrome plurimetabólico, en el que destacan la obesidad, la resistencia a la acción periférica de la insulina y la hiperlipidemia (principalmente la hipertrigliceridemia) (8) como generadores de un desequilibrio de la homeostasis sobre el que el estrés oxidativo puede incidir y producir la progresión de la enfermedad $(15,16)$.

No existe hasta el momento ningún parámetro clínico, analítico, histológico, u otro que nos permita prever el curso evolutivo y los tratamientos hasta hoy probados, se encuentran en fases experimentales además de no haberse realizado estudios correctamente diseñados o con la muestra suficientemente amplia como para tener datos concluyentes sobre su eficacia $(2,6,12,17)$.

\section{Bibliografía}

1. Ludwig J, Viggiano TR, McGill DB, Ott BJ. Nonalcooholic steatohepatitis. Mayo Clinic experiences with a hitherto unnamed disease. Mayo Clinic Proc 1980; 55: 434-8.

2. Gerardo Clemente Ricote y Carmelo García Monzón. Estado actual de la esteatohepatitis no alcohólica. Med Clin (Barc) 2003; 121: 102-8.

3. Ludwig J, McGill DB, Lindor KD. Review: nonalcoholic steatohepatitis. J Gastroenterol Hepatol 1997; 12: 398-403.

4. Tominaga K, Kurata JH, Chen YK, et al. Prevalence of fatty liver in Japanese children and relationship to obesity: an ultrasonographic survey. Dig Dis Sci 1995; 40: 2002-9.

5. Paul Angulo, M. D. Review: nonalcoholic fatty liver disease. N Engl J Me 2003; 346: 1221-31.

6. Andrea E. Reid. Nonoalcoholic steatohepatitis. Gastroenterology 2001; 121: 710-23.

7. Pagano G, Pacini G, Musso G, Gambino R, Mecca F, Depetris N, et al. Nonalcoholic steatohepatitis, insulin resistance and metabolic syndrome: Further evidence for an etiologica association. Hepatology 2002; 35: 367-72.

8. Nimer Assy, Kelly Kaita, David Mymin, Clifford Levy, Barry Rosser and Gerald Minuk. Fatty infiltration of liver in hyperlipidemic patients. Dig Dis Sci 2000; 45: 1929-34.

9. Farrell GC. Steatohepatitis. En Farrell GC, editor. Drug induced liver disease. Edimburgh: Churchill Livingstone, 1995. p. 431-8.
10. Poonawala A, Nair SP, Thuluvath PJ. Prevalence of obesity and patients with cryptogenic cirrhosis: a case-conrol study. Hepatology 2000; 32 689-92.

11. Teli MR, James OFW, Buró AD, Bennet MK, Day CP. The natural history of nonalcoholic fatty liver: a follow up study. Hepatology 1995; 22: 1714-19.

12. Brent A, Neuschwander-Tetri and Stephen H. Caldwell. Nonalcoholic steatohepatitis: summary of an AASLD single topic conference. Hepatology 2003; 37 (5); 1203-19.

13. Matteoni C, Younossi Z, Gramlixh T, Bopari N, Liu Y, McCullough A. Nonalcoholic fatty liver disease: a spectrum of clinical ansd pathological severity. Gastroenterology 1999; 116: 1413-19.

14. Brunt EM, Janney CG, DiBisceglie AM, Neusxhwander-Tetri BA, Bacon BR. Nonalcoholic eteatohepatitis: a proposal for grading and staging the histological lesions. Am Gastroenterol 1999; 94: 2467-74.

15. Day CP, James OLW. Steatohepatitis: a tale of two hits?. Gastroenterology 1998; 114: 842-5.

16. Giulio Marchesini, Elisabetta Bugianesi, et al. Nonalcoholic fatty liver, steatohepatitis, and the metabolic síndrome. Hepatology 2003; 37 (4): 917-23.

17. Kapil Mehta, David H, Van Thiel et al. Nonalcoholic fatty liver disease: pathogenesis and the role of antioxidants. Nutrition Grand Rounds 2002; 60 (9): 289-93. 